\title{
Does the Catford drum give an accurate assessment of acuity?
}

\author{
J. ATKINSON, O. BRADDICK, E. PIMM-SMITH, L. AYLING, AND R. SAWYER \\ From the Kenneth Craik Laboratory, Department of Experimental Psychology, University of Cambridge, \\ Downing Street, Cambridge
}

SUMmaRY Adult emmetropes and myopes were tested with the Catford drum and the results compared with subjective (Landolt $C$ ) acuity. For the emmetropes the Catford drum was found to overestimate visual acuity by a factor of approximately 4 . For myopes, and emmetropes viewing through plus lenses, the discrepancies were much larger. Since the Catford drum not only overestimates acuity but will do so by a factor which varies for different visual disorders, caution is needed in clinical interpretation of results obtained with it.

Catford and Oliver ${ }^{1}$ described an instrument for the objective determination of visual acuity. It consisted of a motor driven drum which could display black dots of various sizes in oscillatory motion against a white background. Acuities were determined by finding the smallest dot which could induce following oscillatory eye movements in the patient. The instrument is commercially available and is widely used in the eye clinics of British hospitals, particularly for assessing the acuity of infants and young children.

Catford and Oliver state that 'The targets are comparable to the equivalent Snellen acuity at 6 metres'. To validate the instrument's scale of measurement they compared acuities measured with it with subjective Snellen acuities for a group of normal observers with acuity progressively reduced with neutral density filters and for a group of amblyopic children. For both groups they report good agreement between the 2 acuity measures, especially for acuities better than $6 / 60$.

However, Catford and Oliver's paper (their Fig. 4) and measurement of the actual targets on the drum make it clear that the spot diameter on the drum for given acuity is equal to the overall size of the Snellen letter for the corresponding acuity. For example, the ' $6 / 60$ ' spot is $9 \mathrm{~mm}$ in diameter and therefore subtends $50 \mathrm{~min}$ arc at the patient's eye when viewed at the recommended distance of $60 \mathrm{~cm}$. The significant dimension of a Snellen letter for determining visual resolution is usually supposed to be the stroke width, which is one-fifth of the letter height. On the 6/60

Correspondence to Dr J. Atkinson, Psychological Laboratory, Downing Street, Cambridge CB2 3EB. letters this stroke width would be not $50 \mathrm{~min}$ arc but $10 \mathrm{~min}$ arc, and $50 \mathrm{~min}$ arc strokes would in fact be resolved by a patient with an acuity of less than $6 / 180$ $(6 / 180$ is equivalent to a stroke width of $30 \mathrm{~min}$ arc). A similar ratio would be expected for the other target sizes. Furthermore, the dimension of the smallest dot that can be detected does not necessarily correspond to the smallest dimension that can be resolved in, for instance, separating the strokes of a Snellen letter. Theoretically the detection of a small dark spot is primarily a test of contrast detection rather than of resolution, and empirically the detection of spots as small as $14 \mathrm{sec}$ has been demonstrated, ${ }^{2}$ even though the smallest detail that can be resolved is $30-60 \mathrm{sec}$ arc. Because the $6 / 6$ Catford target is larger than the resolution limit (which is itself larger than the limit for detecting spots) it would be expected that the $6 / 6$ Catford target would be detectable by individuals with acuity markedly lower than $6 / 6$.

This theoretical argument receives some support from the work of Khan et al. ${ }^{3}$ They used both the Catford drum and subjective measurement to assess acuity both for normal subjects with acuity reduced by filters and by convex lenses, and for patients with various ocular lesions. They found that the Catford drum overestimated acuity compared with subjective assessment in almost every case, although the relationship between the 2 figures differed between their different groups of observers.

If the results of Catford drum tests in the clinic are to be sensibly interpreted, it is important to know what relation such scores do bear to the true acuity. We have therefore compared the visibility of Catford 
Table 1 Comparison of acuities at far on Catford drum and Landolt $C$ for emmetropic eyes

\begin{tabular}{llllll}
\hline Subject & Eye & $\begin{array}{l}\text { Limiting distance } \\
\text { for detection } \\
\text { of Catford 6/6 } \\
\text { target (cm) }\end{array}$ & $\begin{array}{l}\text { Equivalent } \\
\text { acuity }\end{array}$ & $\begin{array}{l}\text { Landolt } C \\
\text { acuity on } \\
\text { Telebinocular } \\
\text { at far }\end{array}$ & $\begin{array}{l}\text { Ratio of } \\
\text { Catford to } \\
\text { Landolt } C \\
\text { acuity }\end{array}$ \\
\hline 1 & L & 290 & $6 / 1 \cdot 2$ & $6 / 4 \cdot 5$ & $3 \cdot 7$ \\
2 & L & 295 & $6 / 1 \cdot 2$ & $6 / 4 \cdot 5$ & $3 \cdot 7$ \\
& R & 270 & $6 / 1 \cdot 3$ & $6 / 4 \cdot 5$ & $3 \cdot 5$ \\
3 & L & 340 & $6 / 1 \cdot 0$ & $6 / 4 \cdot 5$ & $4 \cdot 5$ \\
& R & 400 & $6 / 0 \cdot 9$ & $6 / 4 \cdot 5$ & $5 \cdot 0$ \\
4 & L & 285 & $6 / 1 \cdot 3$ & $6 / 4 \cdot 5$ & $3 \cdot 5$ \\
& R & 275 & $6 / 1 \cdot 3$ & $6 / 4 \cdot 5$ & $3 \cdot 5$ \\
5 & L & 215 & $6 / 1 \cdot 7$ & $6 / 9$ & $5 \cdot 3$ \\
6 & L & 265 & $6 / 1 \cdot 4$ & $6 / 4 \cdot 5$ & $3 \cdot 2$ \\
& R & 265 & $6 / 1 \cdot 4$ & $6 / 4 \cdot 5$ & $3 \cdot 2$ \\
7 & L & 325 & $6 / 1 \cdot 1$ & $6 / 4 \cdot 5$ & $4 \cdot 1$ \\
& R & 390 & $6 / 0 \cdot 9$ & $6 / 4 \cdot 5$ & $5 \cdot 0$ \\
8 & L & 300 & $6 / 1 \cdot 2$ & $6 / 4 \cdot 5$ & $3 \cdot 8$ \\
& R & 360 & $6 / 1 \cdot 0$ & $6 / 4 \cdot 5$ & $4 \cdot 5$ \\
9 & L & 260 & $6 / 1 \cdot 4$ & $6 / 4 \cdot 5$ & $3 \cdot 2$ \\
& R & 270 & $6 / 1 \cdot 3$ & $6 / 9$ & $6 \cdot 9$ \\
10 & L & 365 & $6 / 1 \cdot 0$ & $6 / 4 \cdot 5$ & $4 \cdot 5$ \\
& R & 360 & $6 / 1 \cdot 0$ & $6 / 4 \cdot 5$ & $4 \cdot 5$ \\
11 & L & 265 & $6 / 1 \cdot 4$ & $6 / 6$ & $4 \cdot 3$ \\
& R & 275 & $6 / 1 \cdot 3$ & $6 / 6$ & $4 \cdot 6$ \\
12 & L & 250 & $6 / 1 \cdot 4$ & $6 / 4 \cdot 5$ & $3 \cdot 2$ \\
& R & 260 & $6 / 1 \cdot 4$ & $6 / 4 \cdot 5$ & $3 \cdot 2$ \\
13 & L & 260 & $6 / 1 \cdot 4$ & $6 / 4 \cdot 5$ & $3 \cdot 2$ \\
& R & 260 & $6 / 1 \cdot 4$ & $6 / 4 \cdot 5$ & $3 \cdot 2$ \\
\hline & & & & & \\
\hline
\end{tabular}

drum targets with subjective acuity for a group of emmetropic and myopic observers whose acuities could be reliably subjectively determined.

\section{Materials and methods}

Observers were adults (aged 20-35 years) in the university of Cambridge. The data reported are from 24 emmetropic eyes, and 16 eyes with myopia of $-3.5 \mathrm{D}$ or greater, from a total of 23 individuals. All observers were tested without their spectacle correction, each eye separately.

Subjects were first shown the $6 / 6$ target of the Catford drum (with the motor operating at speed setting 6) at the standard viewing distance of $60 \mathrm{~cm}$ and luminance of $35 \mathrm{~cd} / \mathrm{m}^{2}$. Any observer who reported that he or she could not see the target, and for whom no eyes movements were visible to a tester, was tested with progressively larger Catford targets, and the smallest target which they could detect was determined. Observers who could detect the 6/6 target were asked to walk away from the drum until they reached the point at which the $6 / 6$ target was only just visible, and then to walk in from a greater distance until they reached the distance at which it just became visible again. These 2 distances were recorded and a mean calculated.

In all testing with the Catford drum a tester watched the observer's eye movements from a near distance (about $60 \mathrm{~cm}$ ) and reported whether movements following the oscillation of the target occurred.
All emmetropic subjects were tested with the Catford 6/6 target at $60 \mathrm{~cm}$, wearing a graded series of plus lenses. In each case the maximum lens power was found for which the $6 / 6$ target was still just detectable and the eye movement still visible.

Subjective acuity was tested with the near and far acuity cards in the Keystone Telebinocular (luminance $30 \mathrm{~cd} / \mathrm{m}^{2}$ ). These cards have Landolt C targets from 20/200 to 20/15. For comparison with Catford measurements made at $60 \mathrm{~cm}$ the near acuity card was used at the 1.6 dioptre setting of the Telebinocular. An appropriate correction was made to allow for the fact that the dimensions of the acuity targets are designed for viewing at the closer 2.5 dioptre setting. Observers, for whom the maximum plus lens allowing detection of the Catford target at 60 $\mathrm{cm}$ had been determined, were tested on the 1.6 dioptre setting on the Telebinocular wearing that plus lens. All observers were also tested on the far acuity card in the Telebinocular (at the $0 \cdot 25$ dioptre setting).

\section{Results}

In every case when observers reported that they could detect a Catford target oscillatory following eye movements were visible, and such movements disappeared when the observer reported that the target was invisible. We conclude that in normal adults there is no important difference between the detectability of the Catford targets and their ability to elicit eye movements.

The results of the tests are given in Tables 1,2, and

Table 2 Catford drum measurements on 24 emmetropic eyes

\begin{tabular}{|c|c|c|c|}
\hline \multicolumn{2}{|c|}{ Subject } & \multirow{2}{*}{$\begin{array}{l}\begin{array}{l}\text { Maximum }+ \\
\text { lens for Catford } 6 / 6\end{array} \\
+4 \mathrm{D}\end{array}$} & \multirow{2}{*}{$\begin{array}{l}\begin{array}{l}\text { Landolt } C \\
\text { acuity for same }+ \text { lens }\end{array} \\
\text { Below } 6 / 60\end{array}$} \\
\hline 1 & $\mathbf{L}$ & & \\
\hline 2 & L & $+4 \mathrm{D}$ & $6 / 60$ \\
\hline & $\mathbf{R}$ & $+4 \mathrm{D}$ & $6 / 60$ \\
\hline 3 & L & $+5 \mathrm{D}$ & Below 6/60) \\
\hline & $\mathbf{R}$ & $+6 \mathrm{D}$ & Below 6/60 \\
\hline 4 & L & $+6 \mathrm{D}$ & Below 6/60 \\
\hline & $\mathbf{R}$ & $+4 \mathrm{D}$ & Below 6/60 \\
\hline 5 & L & $+4 \mathrm{D}$ & $6 / 60$ \\
\hline 6 & $\mathbf{L}$ & $+5 \mathrm{D}$ & Below 6/60 \\
\hline & $\mathbf{R}$ & $+5 \mathrm{D}$ & Below $6 / 60$ \\
\hline 7 & L & $+6 \mathrm{D}$ & Below 6/60 \\
\hline & $\mathbf{R}$ & $+5 \mathrm{D}$ & Below 6/60 \\
\hline 8 & L & $+5 \mathrm{D}$ & Below $6 / 60$ \\
\hline & $\mathbf{R}$ & $+5 \mathrm{D}$ & Below 6/60 \\
\hline 9 & $\mathbf{L}$ & $+3 \mathrm{D}$ & $6 / 60$ \\
\hline & $\mathbf{R}$ & $+3 \mathrm{D}$ & $6 / 60$ \\
\hline 10 & $\mathbf{L}$ & $+6 D$ & Below 6/60 \\
\hline & $\mathbf{R}$ & $+7 \mathrm{D}$ & Below 6/60 \\
\hline 11 & L & $+5 \mathrm{D}$ & Below 6/60 \\
\hline & $\mathbf{R}$ & $+5 \mathrm{D}$ & Below 6/60 \\
\hline 12 & L & $+4 D$ & Below 6/60 \\
\hline & $\mathbf{R}$ & $+4 D$ & Below 6/60 \\
\hline 13 & $\mathbf{L}$ & $+4 D$ & $6 / 60$ \\
\hline & $\mathbf{R}$ & $+4 \mathrm{D}$ & $6 / 60$ \\
\hline
\end{tabular}


Table 3 Comparison of acuities at $60 \mathrm{~cm}$ on Catford Drum and Landolt $C$ for myopic eyes

\begin{tabular}{rllll}
\hline Subject & $\begin{array}{l}\text { Refraction } \\
(D)\end{array}$ & $\begin{array}{l}\text { Catford acuity } \\
(\text { at } 60 \mathrm{~cm})\end{array}$ & $\begin{array}{l}\text { Landolt Cacuity } \\
(\text { at } 60 \mathrm{~cm})\end{array}$ \\
\hline 1 & $\mathrm{~L}$ & $-3 \cdot 75$ & $6 / 6$ & $6 / 60$ \\
2 & $\mathrm{~L}$ & $-3 \cdot 7.5$ & Above $6 / 6$ & $6 / 15$ \\
& $\mathrm{R}$ & $-4 \cdot 0$ & $6 / 9$ & $6 / 26$ \\
3 & $\mathrm{~L}$ & $-7 \cdot 5$ & $6 / 12$ & Below $6 / 60$ \\
& $\mathrm{R}$ & $-7 \cdot 75$ & $6 / 12$ & Below $6 / 60$ \\
4 & $\mathrm{~L}$ & $-5 \cdot 0$ & $6 / 9$ & Below $6 / 60$ \\
& $\mathrm{R}$ & $-5 \cdot 0$ & $6 / 9$ & Below $6 / 60$ \\
5 & $\mathrm{~L}$ & $-5 \cdot 0$ & Above $6 / 6$ & Below $6 / 60$ \\
& $\mathrm{R}$ & $-5 \cdot 5$ & Above $6 / 6$ & Below $6 / 60$ \\
6 & $\mathrm{~L}$ & $-7 \cdot 0$ & $6 / 12$ & Below $6 / 60$ \\
& $\mathrm{R}$ & $-7 \cdot 0$ & $6 / 12$ & Below $6 / 60$ \\
7 & $\mathrm{R}$ & $-3 \cdot 5$ & $6 / 6$ & $6 / 52$ \\
8 & $\mathrm{~L}$ & $-6 \cdot 5$ & $6 / 12$ & Below $6 / 60$ \\
& $\mathrm{R}$ & $-6 \cdot 0$ & $6 / 12$ & Below $6 / 60$ \\
9 & $\mathrm{R}$ & $-3 \cdot 5$ & Above $6 / 6$ & Below $6 / 60$ \\
10 & $\mathrm{~L}$ & $-3 \cdot 5$ & Above $6 / 6$ & $6 / 52$ \\
\hline
\end{tabular}

3. All measures of acuity have been converted to 6 metre notation for ease of comparison.

Column 3 of Table 1 shows for emmetropic eyes the maximum distance at which the Catford drum 6/6 target could be detected, and this is converted to an acuity measure in column 4 on the basis that detection of this target at $60 \mathrm{~cm}$ corresponds to $6 / 6$ acuity. For example $6 / 1.0$ in column 4 means that for that individual the 6/6 Catford drum target was still just visible at 6 times the $60 \mathrm{~cm}$ distance, i.e., $360 \mathrm{~cm}$. In column 5 the Landolt $C$ acuity has been recorded and in column 6 a ratio of the 2 acuities is given, i.e., a ratio of the denominators of columns 4 and 5. Thus for subject 1 this ratio is $4 \cdot 5 / 1 \cdot 2=3 \cdot 7$. Table 2 shows the second measure made on the same observers where the plus lens power necessary to eliminate a response to the $6 / 6$ Catford drum target at $60 \mathrm{~cm}$ was measured for each observer. The same lens was then worn for a measurement of Landolt $\mathrm{C}$ acuity. For most subjects acuity was poorer than $6 / 60$ with these lenses. Table 3 shows the Catford acuity and Landolt $\mathrm{C}$ acuity at $60 \mathrm{~cm}$ for 16 myopic eyes of $-3.5 \mathrm{D}$ or more, tested without their spectacle corrections. All of these observers had acuities of 6/12 or better on the Catford drum and very much lower acuities on the Landolt $\mathrm{C}$ test.

In every case we find that the figures based on the Catford drum values considerably overestimate the visual acuity as subjectively determined with Landolt $\mathrm{C}$ targets under equivalent conditions. However, 2 different patterns of results emerge depending on whether the measurements are made on emmetropes in distant vision or on persons whose vision is degraded by refractive errors, in the form either of natural myopia or of plus lenses. For the emmetropes the Catford drum overestimates visual acuity by a factor of approximately 4 . However, for myopes and for emmetropes viewing through plus lenses the dis- crepancy is much larger. The plus lens that just permitted detection of the $6 / 6$ spot at $60 \mathrm{~cm}$ produced a Landolt C acuity of $6 / 60$ or worse in all our emmetropic observers at the equivalent optical distance. Thus for these cases the ratio of the acuity measures is at least 10 . For most of the myopic persons it was similarly possible to give only a minimum estimate of the ratio, either because they could detect the ' $6 / 6$ ' Catford target readily at $60 \mathrm{~cm}$. or because they were unable to resolve the $6 / 60$ Landolt $\mathrm{C}$, or both.

\section{Discussion}

OPTICAL DEFOCUS AND DISTANCE VIEWING COMPARED

The difference between these 2 sets of results is understandable if we consider the 2 optical manipulations involved. In what follows we attempt to express qualitatively a mathematical argument concerning the relationship between the spatial frequency spectrum of a dot target at various distances and the modulation transfer function of the focused or defocused eye. The effect of optically defocusing a target of fixed angular size is greatly to degrade the fine detail information. However, the contrast of larger scale features of the pattern is reduced only by a relatively small amount. Thus with a considerable degree of defocus (such as that produced by $+5 \mathrm{D}$ lenses on the ' $6 / 6$ ' spot at $60 \mathrm{~cm}$ ) a spot is still visible as a broad, faint, low contrast blur. The same defocus of a Landolt $\mathrm{C}$ target may leave the presence of the target quite detectable but make it impossible to resolve the fine detail of the gap. Thus in these circumstances the detection of the Catford target is not a good indicator of visual acuity in the sense of sharp resolution of detail.

However, when an emmetrope moves away from a target, both fine detail and larger scale features are scaled down proportionately in the angle they subtend at the eye. The detectability of both will be reduced. Therefore, even though detection of the Catford target may not depend on the ability to resolve fine detail, the ability to detect the contrast in the target will vary with distance in an approximately similar manner as the detail resolution. In this case, then, even though the Catford values may not provide a quantitatively correct estimate of acuity, they may be converted into a reasonable estimate by a suitable scaling factor. Our data suggest that if the denominator of the fraction in the acuity notation is multiplied by 4 (e.g., $6 / 6$ becomes $6 / 24$, and so on) values from the Catford drum become more realistic.

IMPLICATIONS FOR CLINICAL ASSESSMENT The usefulness of Catford measurements in clinical 
assessment of acuity will depend on the origin and nature of any defect of acuity. If acuity loss is due to refractive error, contrast detection may still be relatively good and the response to 'high-acuity' Catford targets may be quite misleading. This will also be true of any other disorder which affects the resolution of fine detail more severely than the detection of contrast on the larger scale. However, if the nature of the visual defect is such that contrast detection and detail resolution are degraded together, the patient's situation may be more analogous to the emmetrope viewing the target at a distance and the value taken from the Catford drum, multiplied by our suggested correction factor of 4 , may provide a reasonable indication of acuity.

This argument implies that it is hazardous to place any interpretation on readings taken from the Catford drum if the origin of any suspected acuity deficit is unknown; indeed it may not be possible to interpret the readings even in the case of known pathology. Different disorders vary in whether they affect resolution, contrast detection, or both. ${ }^{45}$ In the case of amblyopia a wide variety of patterns of deficit have been found in different patients, and any differences in the aetiology of contrast loss and resolution loss are not yet understood. ${ }^{6-8}$

Many ophthalmologists and orthoptists place little weight on the absolute acuity values obtained with the Catford drum, but regard it as a valuable indicator of any difference in acuity between the 2 eyes of the same patient. In using the instrument this way one should be aware of its insensitivity to quite substantial acuity losses. Under the conditions where it is most sensitive (cases analogous to our emmetropes viewing at distance) the smallest spot available on the drum will not distinguish $6 / 6$ from $6 / 24$ acuity, so this interocular difference would go undetected. This problem could be a great deal worse when there is a refractive difference between the eyes (or some other disorder that analogously produced a loss of detail resolution but not a proportionate loss of contrast sensitivity). In such instances the smallest target can be detected with vision that may be worse than $6 / 60$. It follows that 2 eyes, one with $6 / 6$ and one with $6 / 60$ vision, may both display similar following responses to the ' $6 / 6$ ' Catford target. Expressed another way, up to 5 dioptres of refractive error allow the ' $6 / 6$ ' target to be detected and followed; therefore a 5 dioptre difference in refraction between the eyes could exist and both eyes would still show following of the ' $6 / 6$ ' target.

The most common use of the Catford drum is in the assessment of children under 2 years of age. It might be argued that the inability to measure high levels of acuity is not relevant for this age group. While we have learned much about the early development of acuity, ${ }^{910}$ we still lack reliable normative data for the period between 6 months and 2 years. However, a number of recent studies ${ }^{11-13}$ have suggested that acuities of at least 6/9 may be reached around 6 months to 1 year. Clinical practitioners should therefore be aware of the need for a measuring instrument that will assess the highest levels of acuity even in such young children.

\section{POSSIBLE IMPROVEMENTS TO THE CATFORD} DRUM

Our criticisms of the Catford drum are based on the use of a single spot as an acuity target and on the nominal acuity values assigned to these targets. We believe that in principle the use of an ocular following response to repetitive movement of a discrete target. as proposed by Catford and Oliver, is a robust method which offers one of the best chances of rapid and reliable acuity assessment in children aged between 6 months and about $2 \frac{1}{2}$ years.

To give a true measure of acuity a target is needed which when viewed with blurred vision does not become a diffuse but still detectable spot. Instead blur should make the target actually invisible against its background. This will happen if the target consists of areas both brighter and darker than the background. arranged so that the average luminance of the target area is equal to that of the surrounding area. One example of such a target is an area of grating of light and dark bars, surrounded by an intermediate grev. This will provide the purest test of acuity if the grating fades smoothly into the uniform background. We are currently undertaking trials using targets of this kind to induce following movements in infants aged 9-12 months, and hope that it may be feasible to mount a graded series of such patterns as targets on the Catford drum. With this modification we hope that this ingenious instrument will yield more accurate measurements of developing visual acuity in infants and young children.

This work was supported by the Medical Research Council.

\section{References}

1 Catford GV. Oliver A. Development of visual acuity. Arch Dis Child 1973; 48: 47-50.

2 Riggs LA. Visual acuity. In: Graham $\mathrm{CH}$. ed. Vision and Visual Perception. New York: Wilev. 1966.

3 Khan SG. Chen KF. Frenkel M. Subjective and objective visual acuity testing techniques. Arch Ophthalmol 1976; 94: 2086-91.

4 Bodis-Wollner I. Diamond SP. The measurement of spatial contrast sensitivity in cases of blurred vision associated with cerebral lesion. Brain 1976; 99: 695-710.

5 Arden GB. The importance of measuring contrast sensitivity in cases of visual disturbance. Br J Ophthalmol 1978: 62: 198-2(19.

6 Hess RF. Howell ER. The threshold contrast sensitivity function in strabismic amblyopia: evidence for a two type classification. Vision Res 1977: 17: 1049-55. 
7 Levi M. Harwerth RS. Spatio-temporal interaction in anisometropic and strabismic amblyopia. Invest Ophthalmol Visual Sci 1977; 16: 90-5.

8 Hess RF. Contrast sensitivity assessment of functional amblyopia in humans. Trans Ophthalmol Soc UK 1980; 99: 391-7.

9 Dobson V. Teller DY. Visual acuity in human infants: a review and comparison of behavioral and electrophysiological studies. Vision Res 1978; 18: 1469-83.

10 Atkinson J, Braddick O. Acuity, contrast sensitivity, and accommodation in infancy. In: Aslin RN, Alberts JR, Peterson MR, eds. The Development of Perception, New York: Academic Press, 1981.

11 Marg E, Freeman DN, Peltzman P, Goldstein PJ. Visual acuity development in human infants: evoked potential measurements. Invest Ophthalmol Visual Sci 1976; 15: 150-3.

12 Harris L. Atkinson J, Braddick O. Visual contrast sensitivity of a 6 month-old infant measured by the evoked potential. Nature 1976; 264: 570-1.

13 Sokol S. Measurement of infant visual acuity from pattern reversal evoked potentials. Vision Res 1978; 18: 33-9. 